\title{
Semi-Feedback for the Binary Multiplying Channel
}

\author{
J. Pieter M. Schalkwijk, and Hendrik B. Meeuwissen \\ Eindhoven University of Technology, Department of Electrical Engineering, Group of Information and Communication \\ Theory, P.O.Box 513, 5600 MB Eindhoven, The Netherlands
}

I. Introduction

In his 1961 paper [1] on Two-Way Channels (TWC's) Shannon derived the so-called inner and outer bound region. For a TWC without feedback the outer bound coincides with the inner bound. As a consequence, the capacity region of a TWC without feedback is equal to its inner bound. Furthermore, Shannon showed in his initiating paper that for the Binary Multiplying Channel (BMC) the inner and outer bound are different. The equal rate point $\left(R_{1}, R_{2}\right)$ on the inner bound of the BMC satisfies $R_{1}=R_{2}=R=0.61695$, while the equal rate point $\left(R_{1}, R_{2}\right)$ on the outer bound satisfies $R_{1}=R_{2}=R=0.69424$

In 1979 Dueck [2] proved the existence of a TWC with full-feedback for which the capacity region is in excess of its inner bound region. Next, in 1982 Schalkwijk [3] constructed a coding strategy for the BMC that achieves $R_{1}=R_{2}=R=$ 0.61914 outside of the inner bound region. Then, in 1983 Schalkwijk [4] further extended the achievable rate region of the $\mathrm{BMC}$ towards $R_{1}=R_{2}=R=\mathbf{0 . 6 3 0 5 6}$ by a technique called bootstrapping. Both the 1982 and the 1983 strategy are based on the progressive subdivision of a unit square of message points $\Theta=\left(\Theta_{1}, \Theta_{2}\right)$ using feedback at both terminals.

In 1980 Dueck [5] also proved the existence of a TWC with feedback at one terminal (semi-feedback) for which the capacity region is in excess of its inner bound region. However, the BMC with semi-feedback was believed to have a capacit region that coincides with the inner bound region. Neverthe less, a semi-strategy has been constructed for the BMC that operates beyond the inner bound region. The semi-strategy is based on both the new message percolation technique [6] and the old bootstrapping technique.

II. An achievable rate region

The BMC has two binary inputs $X_{1}$ and $X_{2}$, and one common binary output $Y$, defined by $Y_{1}=Y_{2}=Y=$ $X_{1} X_{2}$. Encoder 1 forms the channel input sequence $X_{1}=$ $\left(X_{1,1} X_{1,2}, \ldots, X_{1, n}\right)$ using both the message $\Theta_{1}$ and the channel outputs $Y_{1}, Y_{2}, \ldots, Y_{n-1}$ that are available because of the feedback link. The channel input sequence $\boldsymbol{X}_{2}=$ $\left(X_{2,1} X_{2,2}, \ldots, X_{2, n}\right)$ of encoder 2 depends on the message $\Theta_{2}$ only. Thus encoder 1 uses a strategy, while encoder 2 uses a code.

The first transmission $X_{i, 1}, i=1,2$, at terminal $i$ can only depend on the message $\Theta_{i}$. Consequently, we need at least two transmissions to improve upon the inner bound. In this section we will show that if message percolation is applied, then exactly two transmissions are needed to outperform the inner bound. The first transmission of the depth 2 semi-strategy is $X_{i, 1}=1, i=1,2$, if $\Theta_{i} \in\left[0, a_{i}\right)$, or $X_{i, 1}=0, i=1,2$ if $\Theta_{i} \in\left[a_{i}, 1\right)$. Whether the message point $\Theta$ is in the resolution product $\left[0, a_{1}\right) \times\left[0, a_{2}\right)$ or in the resolution product $(0,1) \times[0,1)-\left[0, a_{1}\right) \times\left[0, a_{2}\right)$ is not only known to decode 1 and 2 , but also to encoder 1 . Subsequently, we can apply bootstrapping [4]. In that manner we can confine ourselves to the subrectangle $\left[a_{1}-a_{4}, 1\right) \times\left[a_{2}-a_{3}, 1\right)$ without affecting the rate. The dashed lines in the figure are transparent to encoder 2 , because of the absence of feedback.

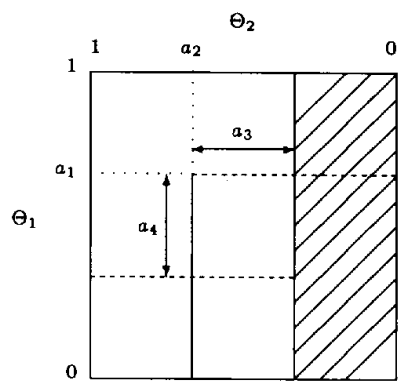

Now suppose that we have a sequence $\left(\boldsymbol{\Theta}_{1}, \boldsymbol{\Theta}_{2}, \ldots, \boldsymbol{\Theta}_{l}\right)$ of length $l$ of message points, such that for each message point $\Theta_{j}, j=1,2, \ldots, l$, in this sequence both the first transmission and the subsequent bootstrapping resolution have been carried out. Then encoder 1 can interchange its messages in the interval $\left[0, a_{1}-a_{4}\right]$ with messages in the interval $\left[a_{1}-a_{4}, a_{1}\right)$. In other words, encoder 1 percolates its messages and constructs a new sequence of message points $\left(\Theta_{1}^{\prime}, \Theta_{2}^{\prime}, \ldots, \Theta_{\ell}^{\prime}\right)$, such that the first $k$ message points in the new sequence are in the rectangle $\left[a_{1}-a_{4}, 1\right) \times\left[a_{2}-a_{3}, 1\right)$. For these $k$ initial members a second transmission is done. Note that the order of the messages is changed at terminal 1 . This is in contrast with one-way communication, where the order of the messages is irrelevant. For $l \rightarrow \infty$ the information theoretical maximal half-sum rate of the new semi-strategy equals 0.61818 in the point $(0.61651,0.61985)$. Thus encoder 1 uses the feedback in this maximum to percolate its messages, such that encoder 2 can transmit at the highest rate.

1] C. E. Shannon, "Two way communication channels," Proceed ings 4th Berkely Symposium an Mathematics, Statistics and Probability, vol. 1, pp. 611-644, 1961.

[2] G. Dueck, "The capacity region of the twc can exceed the inner bound," Information and Control, vol. 40, pp. 258-266, 1979.

[3] J.P.M. Schalkwijk, "The binary multiplying channel - a coding scheme that operates beyond the Shannon inner bound," IEE Transactions on Information Theory, vol. IT-28, no. 1, pp. 107110, Jan. 1982

[4] J.P.M. Schalkwijk, "On an extension of an achievable rate region for the binary multiplying channel," IEEE Transactions on Information Theary, vol. IT-29, no. 3, pp. 445-448, May 1983.

[5] G. Dueck, "Partial feedback for two-way and broadcast channels," Information and Control, vol. 46, pp. 1-15, 1980.

[6] J.P.M. Schalkwijk, "Semi-feedback and message percolation," Proceedings Joint Swedish-Russian International Workshop on Information Theory, vol. 6, pp. 87-91, Mölle, Sweden, 1993. 